\title{
Emergence of a new recombinant Sydney 2012 norovirus variant in Denmark, 26 December 2012 to 22 March 2013
}

J Fonager (fon@ssi.dk) ${ }^{1}$, S Barzinci ${ }^{1}$, T K Fischer ${ }^{1}$

1. Department of Microbiological Diagnostics and Virology, Statens Serum Institut, Copenhagen, Denmark

Citation style for this article:

Fonager J, Barzinci S, Fischer TK. Emergence of a new recombinant Sydney 2012 norovirus variant in Denmark, 26 December 2012 to 22 March 2013 . Euro Surveill. 2013;18(25):pii=20506. Available online: http://www.eurosurveillance.org/ViewArticle.aspx?Articleld=20506

Article submitted on 03 June 2013 /published on 20 June 2013

We report here new recombinants between the norovirus II.4 Sydney 2012 and the II.4 New Orleans 2009 variants. This demonstrates that the II.4 Sydney 2012 variant is undergoing further diversification and suggests a potential for rapid evolution. We also provide primers, which allow the amplification and sequencing of both the current New Orleans 2009 and Sydney 2012 variants and the new II.4 New Orleans 2009/II.4 Sydney 2012 recombinants for more accurate surveillance and transmission tracking.

In the period between December 2012 and April 2013, a selection of norovirus ( $\mathrm{NoV}$ )-positive samples which had been submitted to Statens Serum Institute (SSI) for diagnostic purposes were further characterised by typing sequences of the NoV derived polymerase (pol) and capsid (cap) genes. Based on their characteristics, the pol and cap sequences were respectively assigned to a particular NoV genotype within genogroup I (GI) or genogroup II (GII), including NoV GII, genotype 4 (II.4) variants where applicable. A number of samples tested concomitantly positive for the pol of one NoV variant and the cap of another. For example, the GII, genotype e (II.e) Sydney 2012 NoV pol/II.4 Sydney 2012 NoV cap sequences were co-detected in some samples and in other samples the II.4 New Orleans 2009 NoV pol/II.4 Sydney 2012 NoV cap sequences were co-detected. As this could potentially indicate the presence of recombinant NoV in such samples, primers for a polymerase chain reaction (PCR) assay were designed to investigate if recombinants could be detected in the samples.

\section{Background}

The NoV Sydney 2012 variant has since late 2012 attracted global attention and several countries have reported an associated increase in NoV cases and a rapid evolution of this variant [1-4]. The earlier dominant New Orleans 2009 variant [5] has been replaced by the Sydney 2012 variant in the United States [3]. We provide here evidence for the emergence of recombinant Sydney 2012 variants and present primers to enable the amplification and sequencing of these variants.

\section{New recombinants}

Characterisation of NoV was done by typing of the $\mathrm{NoV}$ pol and cap regions. Typing of the pol region was conducted using a first round reverse transcriptionPCR (RT-PCR) with the primers $N_{32}, N_{32} a$ (forward primers)/NV36 (reverse primer), followed by a second PCR using the primers $\mathrm{NV}_{33}, \mathrm{NV}_{33}$ a (forward primers)/ $\mathrm{NV}_{35}, \mathrm{NV}_{35}$ a (reverse primers) $[6,7]$. The PCR primers in this assay were designed to anneal conserved regions shared by NoV of GI and GII and can amplify both NoV GI and GII viral sequences. Typing of the cap region consisted in a semi-nested PCR for either GI or GII viral sequences. In the first RT-PCR round, the primers $\mathrm{G}_{1} \mathrm{FF} / \mathrm{G}_{1} \mathrm{SKR}$ for $\mathrm{GI}$ or $\mathrm{G}_{2} \mathrm{FB} / \mathrm{G}_{2} \mathrm{SK}$ for GII $[8,9]$ were used, followed by a second round PCR using the primers GIFFN/G1SKR (GI) or GIIFBN/G2SKR (GII) $[9,10]$. This allowed us to retrieve and analyse the pol and cap genetic sequences derived from individual NoV positive samples submitted to SSI for routine diagnostics, and to respectively assign each sequence to a NoV variant. Co-detection in a sample of a pol sequence characteristic of one NoV variant and a cap sequence characteristic of another could suggest possible NoV recombinants.

The first sample with evidence of possible recombination had been sent to SSI on 21 December 2012. In the period from 8 December 2012 to 12 April 2013, a total of 34 samples were typed in both the pol and cap genes and of these, three (9\%) were II.4 New Orleans 2009 in both pol and cap, 10 (29\%) were II.e Sydney 2012 in pol and II.4 Sydney 2012 in cap and 16 (47\%) were II. 4 New Orleans 2009 in pol and II.4 Sydney 2012 in cap, while the remaining five (15\%) were other NoV types (Figure 1).

Interestingly, we did not observe any indications for a recombination of the II.e Sydney 2012 NoV pol and the II.4 New Orleans 2009 NoV cap sequences, which could indicate a strong selection pressure against continued circulation of any NoV variant containing the New Orleans 2009 NoV cap. 


\section{FIGURE 1}

Characterisation of norovirus positive samples by analysing respective polymerase and capsid sequences, Denmark, 08 December 2012-12 April 2013 ( $n=34)$

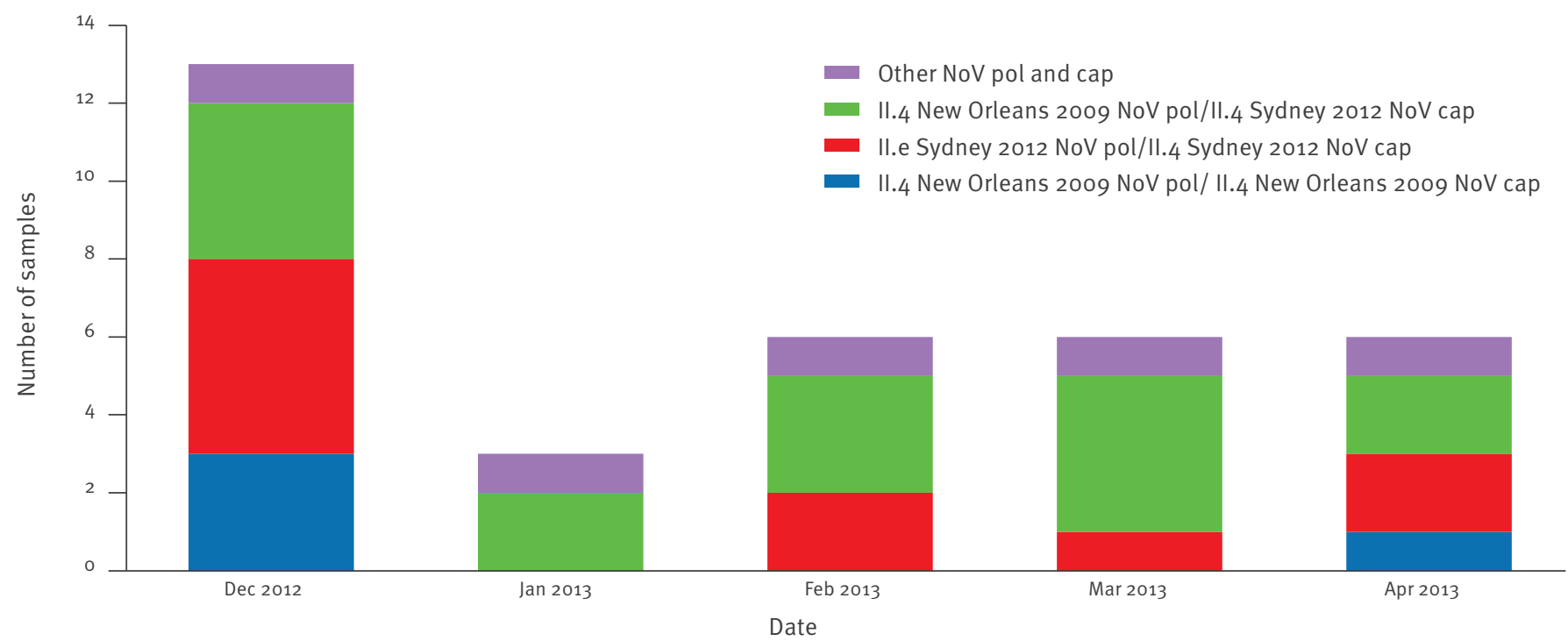

Cap: capsid; NoV: norovirus; pol: polymerase.

The pol and cap type are indicated in the coloured legend.

\section{TABLE}

Primers used for polymerase chain reaction amplification or sequencing of a norovirus contiguous genomic region sequence containing polymerase and capsid regions in order to detect norovirus recombinants

\begin{tabular}{|c|c|c|c|c|c|}
\hline Reaction types & Primer name & $\begin{array}{c}\text { Primer } \\
\text { orientation }\end{array}$ & Primer sequence ( $5^{-}$to $\left.3^{\prime}\right)$ & $\begin{array}{c}\text { Annealing } \\
\text { position }^{a}\end{array}$ & $\begin{array}{l}\text { PCR product size } \\
(\mathrm{bp})\end{array}$ \\
\hline $\begin{array}{l}\text { First } P C R^{b} / \\
\text { sequencing }\end{array}$ & Pol F1 & Forward & CAGAACCACATTTGGCTCAGGTAGTC & 4,371 & 1,325 \\
\hline \multirow{2}{*}{$\begin{array}{l}\text { Second } P C R^{b, c} / \\
\text { sequencing }\end{array}$} & Pol F2 & Forward & GCGACTTCACAATATCAATCAACGAGG & 4,434 & \multirow{2}{*}{846} \\
\hline & Capsid R2 & Reverse & TGTAAACTCTCCACCAGGGGCTTGTAC & 5,280 & \\
\hline
\end{tabular}

PCR: polymerase chain reaction.

a Relative to the II.4 New Orleans 2009 norovirus variant reference sequence (GenBank accession number: GU445325.2).

b The primers for this PCR can equally well amplify a genomic region with the New Orleans 2009 polymerase and capsid sequence, a genomic region with the Sydney 2012 polymerase and capsid sequences, or a recombinant genomic region with the New Orleans 2009 polymerase and the Sydney 2012 capsid sequences.

c The second PCR is optional and can be used for samples with no product after the first PCR. 


\section{FIGURE 2}

Phylogenetic analysis of the contiguous polymerase and capsid sequence regions retrieved from norovirus positive samples, Denmark, 03 January-30 March 2013 (n=21)

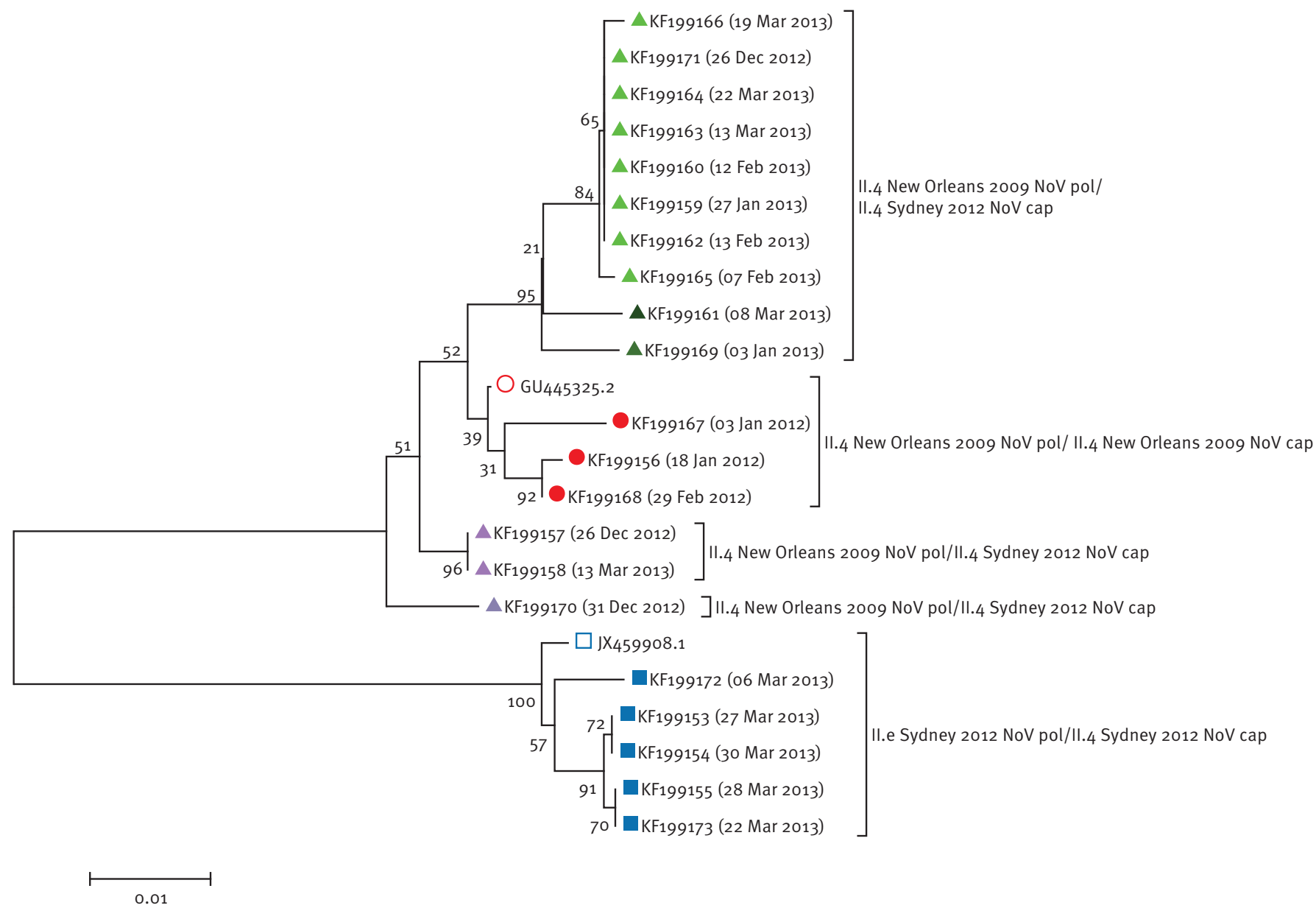

Cap: capsid; NoV: norovirus; pol: polymerase.

Filled icons represent tested samples in this study; outlined icons represent reference sequences.

The tree is a Neighbour Joining tree. Numbers at the nodes indicate supporting bootstrap values obtained from 1,00o replicates. Triangles with the same colour represent sequences differing from each other by no more than five single nucleotide polymorphisms. On the tree leaves the GenBank accession numbers of sequences are shown. When sequences are not reference sequences, the GenBank number is followed by the collection date of the sample from which the sequence was derived.

The pol and cap region types of the sequences (which were determined by typing for non-reference sequences) are shown to the side of the tree. 
Simplot showing the percentage similarity between selected sequences retrieved from norovirus positive samples ( $\mathrm{n}=8$ ) in Denmark and the II.4 New Orleans 2009 reference sequence (GenBank accession number: GU445325.2)

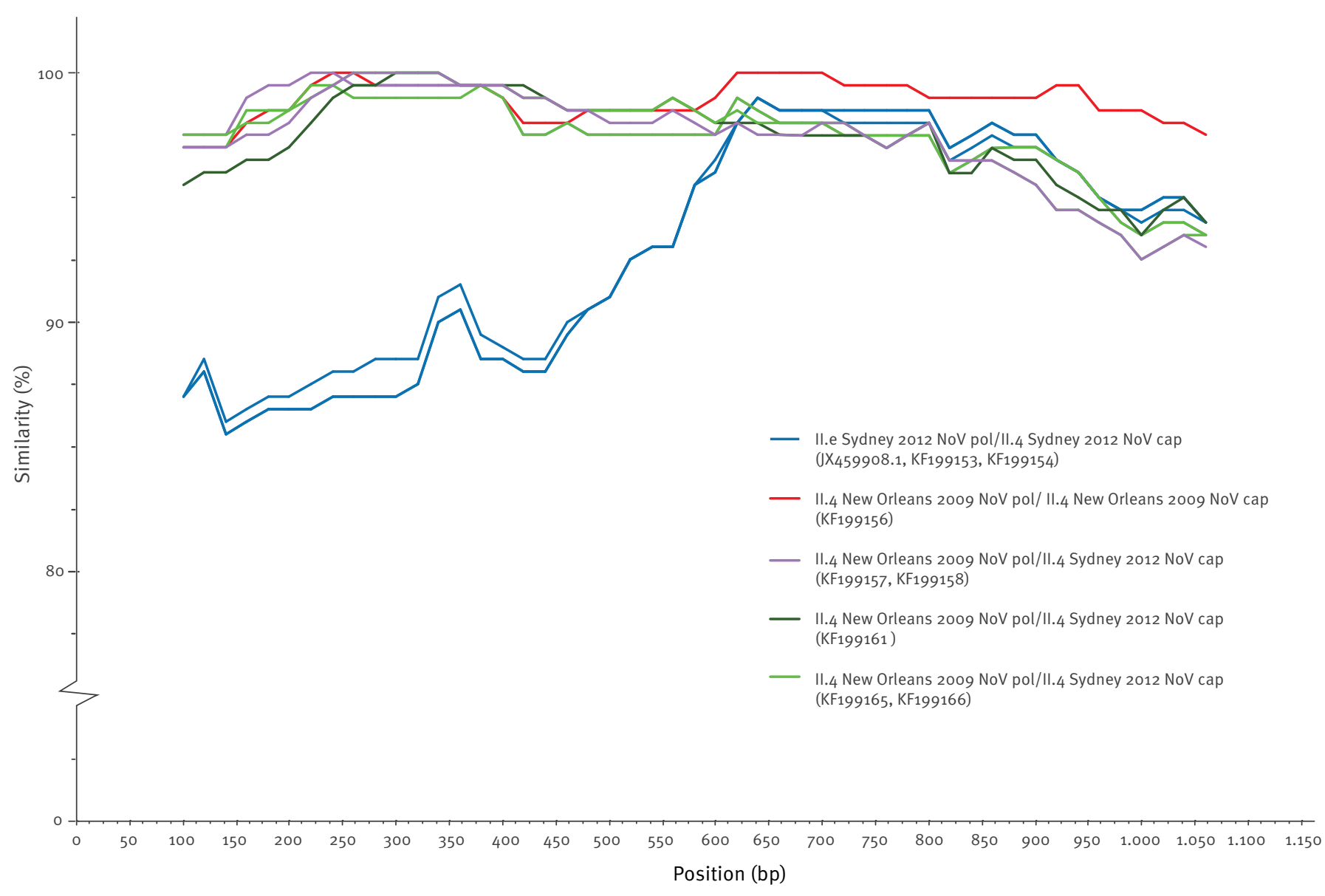

Cap: capsid; NoV: norovirus; pol: polymerase.

The New Orleans 2009 norovirus reference sequence (GenBank accession number: GU445352.2) was used as the query sequence. In the legend, the GenBank accession numbers of the sequences are given for all eight sequences obtained from samples in this study and the Sydney 2012 reference sequence (GenBank accession number: JX 459908.1) as well as the pol and cap type. For the II.4 New Orleans 2009 NoV pol/II.4 Sydney 2012 NoV cap recombinants, the different line colours indicate that these sequences originate from independent recombination events. The Simplot settings were: Window: 200 bp, Step: 20, GapStrip: On.

Since the pol and cap typing produce two non-overlapping sequences, the possibility of patients having a double infection with both the II.4 New Orleans $2009 \mathrm{NoV}$ pol/II.4 New Orleans 2009 NoV cap and the II.e Sydney 2012 NoV pol/II.4 Sydney 2012 NoV cap variants could not be excluded. Therefore, to confirm recombination, we designed a set of nested primers (Table), allowing for amplification and sequencing of a contiguous genomic region containing both parts of the pol and cap regions used for typing, and the intergenic region.

In total, 21 samples, collected in the period from 03 January 2012 to 30 March 2013 were RT-PCR amplified and directly sequenced using the primers in the Table. Typing was initially performed using the typing tool implemented at the National Institute for Public Health and the Environment (RIVM) in the Netherlands [11]. Of the 21 amplified sequences, 19 were long enough to yield sequencing results for both the pol and cap regions, while two yielded sequencing results for the entire pol sequence but were too short to include the complete cap sequence. These two sequences were respectively derived from two samples which had been included in the 34 samples previously typed by independent amplification of the pol and cap sequences. Therefore the missing cap region of the two incomplete sequences could be obtained from the result of this previous typing approach. Phylogenetic analysis was performed on the sequences (Figure 2) using the Neighbour Joining method with Jukes Cantor implemented in Mega 5 [12]. 
According to the phylogenetic tree, the sequences which were typed as II.4 New Orleans 2009 pol/II.4 New Orleans 2009 NoV cap or II.e Sydney 2012 NoV pol/Il.4 Sydney 2012 NoV cap formed clusters with their respective reference sequences, whereas the sequences typed as II.4 New Orleans 2009 NoV pol/ II.4 Sydney NoV 2012 cap did not cluster together with any of these reference sequences. Most (8 of 13) of the recombinant II.4 New Orleans $2009 \mathrm{NoV}$ pol/II.4 Sydney 2012 NoV cap sequences were localised to a single cluster (light green triangles on Figure 2). The samples in this cluster were collected during a period of 86 days (from 26 December 2012 to 22 March 2013) and differ from each other by no more than five single nucleotide polymorphisms (SNPs). These recombinants therefore probably arose from a unique recombinant, circulating in the Danish population during this period, which had been generated from a single recombination event. Another small cluster containing two sequences (pink triangles) and three other unclustered sequences (dark green, green and purple triangles) were distinctively different from the dominant cluster (light green triangles) (Figure 2). These five sequences were all collected during the same time period as the sequences in the majority cluster and probably arose from four individual recombination events. Therefore, this analysis confirms that II.4 New Orleans $2009 \mathrm{NoV}$ pol/II.4 Sydney 2012 NoV cap recombinants have emerged independently and that up to five individual recombination events can be observed among the analysed samples.

Eight selected NoV sequences from this study and the II.e pol/II.4 cap Sydney 2012 reference sequence (GenBank accession number: JX 459908.1) were further analysed over a region of 1,161 nucleotides using Simplot 1.3 [13] with a windows size of 200 and a 20 bp step using the New Orleans 2009 reference sequence (GenBank accession number: GU445325.2) as the query sequence (Figure 3). The selected sequences included two sequences from the study defined as having a II.e Sydney 2012 NoV pol and a II.4 Sydney 2012 NoV cap (Figure 3, in blue), one sequence from the study defined as having a II.4 New Orleans 2009 NoV pol and a II.4 New Orleans 2009 NoV cap (Figure 3, in red) and five sequences with a II.4 New Orleans 2009 NoV pol and a II.4 Sydney 2012 cap. These latter five sequences were chosen as representatives of recombinants which arose from three independent pol/cap recombination events (Figure 3, in dark green, light green and purple). The Simplot shows that all the chosen II.4 New Orleans 2009 NoV pol/II.4 Sydney 2012 NoV cap recombinant sequences (dark green, light green and pink lines) are very similar to the II.4 New Orleans $2009 \mathrm{NoV}$ pol/II.4 New Orleans 2009 NoV cap sequences (red line) in the pol region and a have a cap region similar to II.4 Sydney 2012 NoV cap.

The region around position 600 to 625 (position 5,037 to 5,062 on the II.4 New Orleans 2009 reference sequence (GenBank accession number: GU445325.2), where the similarity between the II.e Sydney 2012 NoV pol/II.4 Sydney 2012 NoV cap sequences (Figure 3, blue lines), and the II.4 New Orleans 2009 query sequence, reaches a maximum, corresponds to the beginning of the II.4 Sydney 2012 NoV cap gene sequence. As this sequence is common to the II.4 New Orleans 2009 NoV pol/II.4 Sydney 2012 NoV cap recombinants (dark green, light green and pink lines) this indicates that recombination has occurred in this region.

\section{Conclusion}

Recombination is a major diversifying factor in the evolution of several NoV genotypes and II.4 variants $[14,15]$ and might result in the emergence of more virulent variants $[16,17]$. We report here the emergence of new recombinants of the Sydney 2012 NoV variant, which shows that this variant is undergoing further diversification. It remains to be determined how virulent the new recombinants will be, including their capability to escape the acquired herd immunity. We also here provide primers, which will allow for identification of both the II.4 New Orleans $2009 \mathrm{NoV}$ pol/II.4 New Orleans 2009 NoV cap and II.e Sydney 2012 NoV pol/II.4 Sydney 2012 NoV cap and the recombinant II.4 New Orleans 2009 NoV pol/II.4 Sydney 2012 NoV cap variants for more accurate surveillance and transmission tracking.

\section{Acknowledgements}

We thank all general practitioners and Clinical Microbiological departments in Denmark, who provided samples for norovirus analysis. Also, we would like to thank Hanne Marie Holt for providing us with a sample from a suspected norovirus outbreak.

Conflict of interest

None declared.

Authors' contributions

J Fonager: Conceived the idea for the study and performed sequence, phylogenetic and Simplot analysis and drafted the first version of the paper, made revisions, and approved the final version of the paper. TK Fischer: Designed the project together with J Fonager, provided constructive comments and revised the first draft of the paper critically and approved the final version of the paper. S Barzinci: Contributed considerably with the laboratory analyses and approved the final version of the paper. 


\section{References}

1. van BJ, Ambert-Balay K, Botteldoorn N, Eden JS, Fonager J, Hewitt J, et al. Indications for worldwide increased norovirus activity associated with emergence of a new variant of genotype II.4, late 2012. Euro Surveill. 2013;18(1):pii=20345. Available from: http://www.eurosurveillance.org/ViewArticle. aspx?Articleld $=20345$

2. Bennett S, MacLean A, Miller RS, Aitken C, Gunson RN. Increased norovirus activity in Scotland in 2012 is associated with the emergence of a new norovirus GII. 4 variant. Euro Surveill. 2013;18(2):pii=20349. Available from: http://www. eurosurveillance.org/ViewArticle.aspx?Articleld=20349 PMid:23324428

3. Centers for Disease Control and Prevention (CDC). Emergence of new norovirus strain GIl.4 Sydney--United States, 2012. MMWR Morb Mortal Wkly Rep. 2013;62(3):55. PMid:23344699

4. Fonager J, Hindbaek LS, Fischer TK. Rapid emergence and antigenic diversification of the norovirus 2012 Sydney variant in Denmark, October to December, 2012. Euro Surveill. 2013;18(9):pii=20413. Available from: http://www. eurosurveillance.org/ViewArticle.aspx?Articleld $=20413$

5. Division of Viral Diseases, National Center for Immunization and Respiratory Diseases, Centers for Disease Control and Prevention. Updated norovirus outbreak management and disease prevention guidelines. MMWR Recomm Rep. 2011;60(RR-3):1-18.

6. Schreier E, Doring F, Kunkel U. Molecular epidemiology of outbreaks of gastroenteritis associated with small round structured viruses in Germany in 1997/98. Arch Virol. 2000;145(3):443-53. http://dx.doi.org/10.1007/ s007050050038 PMid:10795514

7. Oh DY, Gaedicke G, Schreier E. Viral agents of acute gastroenteritis in German children: prevalence and molecular diversity. J Med Virol. 2003;71(1):82-93. http://dx.doi. org/10.1002/jmv.10449 PMid:12858413

8. Kageyama T, Kojima S, Shinohara M, Uchida K, Fukushi S, Hoshino FB, et al. Broadly reactive and highly sensitive assay for Norwalk-like viruses based on real-time quantitative reverse transcription-PCR. J Clin Microbiol. 2003;41(4):154857. http://dx.doi.org/10.1128/JCM.41.4.1548-1557.2003 PMid:12682144 PMCid:153860

9. Kojima S, Kageyama T, Fukushi S, Hoshino FB, Shinohara M, Uchida K, et al. Genogroup-specific PCR primers for detection of Norwalk-like viruses. J Virol Methods. 2002;100(1-2):107-14. http://dx.doi.org/10.1016/S0166-0934(01)00404-9

10. Gallimore CI, Cheesbrough JS, Lamden K, Bingham C, Gray JJ. Multiple norovirus genotypes characterised from an oyster-associated outbreak of gastroenteritis. Int J Food Microbiol. 2005;103(3):323-30. http://dx.doi.org/10.1016/j. ijfoodmicro.2005.02.003 PMid:15967530

11. Kroneman A, Vennema H, Deforche K, v d Avoort H, Penaranda $\mathrm{S}$, Oberste MS, et al. An automated genotyping tool for enteroviruses and noroviruses. J Clin Virol. 2011;51(2):121-5. http://dx.doi.org/10.1016/j.jcv.2011.03.006 PMid:21514213

12. Tamura K, Peterson D, Peterson N, Stecher G, Nei M, Kumar S. MEGA5: molecular evolutionary genetics analysis using maximum likelihood, evolutionary distance, and maximum parsimony methods. Mol Biol Evol. 2011;28(10):2731-9. http://dx.doi.org/10.1093/molbev/msr121 PMid:21546353 PMCid:3203626

13. Lole KS, Bollinger RC, Paranjape RS, Gadkari D, Kulkarni SS Novak NG, et al. Full-length human immunodeficiency virus type 1 genomes from subtype C-infected seroconverters in India, with evidence of intersubtype recombination. J Virol. 1999;73(1):152-60. PMid:9847317 PMCid:103818

14. Eden JS, Tanaka MM, Boni MF, Rawlinson WD, White PA. Recombination within the Pandemic Norovirus GII.4 Lineage. J Virol. 2013;87(11):6270-82. http://dx.doi.org/10.1128/ JVI.03464-12 PMid:23536665

15. Bull RA, Tanaka MM, White PA. Norovirus recombination. Gen Virol. 2007;88(Pt 12):3347-59. http://dx.doi.org/10.1099/ vir.0.83321-o PMid:18024905

16. Mathijs E, Denayer S, Palmeira L, Botteldoorn N, Scipioni A, Vanderplasschen $A$, et al. Novel norovirus recombinants and of GII. 4 sub-lineages associated with outbreaks between 2006 and 2010 in Belgium. Virol J. 2011;8:310. http://dx.doi. org/10.1186/1743-422X-8-310 PMid:21682917 PMCid:3135559

17. Giammanco GM, Rotolo V, Medici MC, Tummolo F, Bonura F, Chezzi C, et al. Recombinant norovirus GII.g/GII.12 gastroenteritis in children. Infect Genet Evol. 2012;12(1):16974. http://dx.doi.org/10.1016/j.meegid.2011.10.021 PMid:22067516 